\title{
Acyl-lipid desaturases and Vipp1 cooperate in cyanobacteria to produce novel omega-3 PUFA-containing glycolipids
}

Leslie B. Poole ${ }^{1,2^{*}}$ (1) Derek Parsonage ${ }^{1,2}$, Susan Sergeant ${ }^{1}$, Leslie R. Miller ${ }^{3,7}$, Jingyun Lee ${ }^{4,5}$, Cristina M. Furdui ${ }^{2,4,5}$ and Floyd H. Chilton $3,5,6^{*}$

\begin{abstract}
Background: Dietary omega-3 ( $n-3)$, long chain (LC-, $\geq 20$ carbons), polyunsaturated fatty acids (PUFAs) derived largely from marine animal sources protect against inflammatory processes and enhance brain development and function. With the depletion of natural stocks of marine animal sources and an increasing demand for n-3 LC-PUFAs, alternative, sustainable supplies are urgently needed. As a result, n-3 18-carbon and LC-PUFAs are being generated from plant or algal sources, either by engineering new biosynthetic pathways or by augmenting existing systems.

Results: We utilized an engineered plasmid encoding two cyanobacterial acyl-lipid desaturases (DesB and DesD, encoding $\Delta 15$ and $\Delta 6$ desaturases, respectively) and "vesicle-inducing protein in plastids" (Vipp1) to induce production of stearidonic acid (SDA, 18:4 n-3) at high levels in three strains of cyanobacteria (10, 17 and 27\% of total lipids in Anabaena sp. PCC7120, Synechococcus sp. PCC7002, and Leptolyngbya sp. strain BL0902, respectively). Lipidomic analysis revealed that in addition to SDA, the rare anti-inflammatory n-3 LC-PUFA eicosatetraenoic acid (ETA, 20:4 n-3) was synthesized in these engineered strains, and 99\% of SDA and ETA was complexed to bioavailable monogalactosyldiacylglycerol (MGDG) and digalactosyldiacylglycerol (DGDG) species. Importantly, novel molecular species containing alpha-linolenic acid (ALA), SDA and/or ETA in both acyl positions of MGDG and DGDG were observed in the engineered Leptolyngbya and Synechococcus strains, suggesting that these could provide a rich source of antiinflammatory molecules.
\end{abstract}

Conclusions: Overall, this technology utilizes solar energy, consumes carbon dioxide, and produces large amounts of nutritionally important n-3 PUFAs and LC-PUFAs. Importantly, it can generate previously undescribed, highly bioavailable, anti-inflammatory galactosyl lipids. This technology could therefore be transformative in protecting ocean fisheries and augmenting the nutritional quality of human and animal food products.

Keywords: Omega-3 polyunsaturated fatty acids, Bioengineering, Nutrition, Aquaculture, Cyanobacteria, Leptolyngbya

\footnotetext{
*Correspondence: Ibpoole@wakehealth.edu; fchilton@email.arizona.edu 1 Department of Biochemistry, Wake Forest School of Medicine, Winston-Salem, NC 27157, USA

${ }^{6}$ Department of Nutritional Sciences and the BIO5 Institute, University of Arizona, Tucson, AZ, USA

Full list of author information is available at the end of the article
}

\begin{abstract}
Background
Eighteen-carbon (18C), omega-3 (n-3) polyunsaturated fatty acids (PUFAs) and particularly n-3 long chain (LC, $\geq 20$ carbons) PUFAs have been shown to exert anti-inflammatory and cardioprotective roles in cardiovascular disease and several inflammatory diseases [1]. Additionally, n-3 LC-PUFAs are essential for early childhood development, and deficiencies of n-3
\end{abstract}

(c) The Author(s) 2020. This article is licensed under a Creative Commons Attribution 4.0 International License, which permits use, sharing, adaptation, distribution and reproduction in any medium or format, as long as you give appropriate credit to the original author(s) and the source, provide a link to the Creative Commons licence, and indicate if changes were made. The images or other third party material in this article are included in the article's Creative Commons licence, unless indicated otherwise in a credit line to the material. If material is not included in the article's Creative Commons licence and your intended use is not permitted by statutory regulation or exceeds the permitted use, you will need to obtain permission directly from the copyright holder. To view a copy of this licence, visit http://creativeco mmons.org/licenses/by/4.0/. The Creative Commons Public Domain Dedication waiver (http://creativecommons.org/publicdomain/ zero/1.0/) applies to the data made available in this article, unless otherwise stated in a credit line to the data. 
LC-PUFAs are associated with mental disorders and cognitive decline [2-5]. Consequently, several health organizations recommend increasing dietary consumption of n-3 PUFAs and LC-PUFAs, resulting in rapidly growing markets for these in functional foods, pharmaceuticals, dietary supplements and infant formulas $[6$, 7].

However, expansions in demand for n-3 PUFAs and n-3 LC-PUFAs have raised vital questions about their sustainability. For example, fish represent the predominant source of n-3 LC-PUFAs; however, wild caught fish are at or beyond exploitable limits, and more than half of fish consumed are farmed [7]. Krill oil as another unsustainable source of n-3 LC-PUFAs exerts even greater strains on the global health of ocean fisheries. Approximately $75 \%$ of the global supply of n-3 LC-PUFAs is currently utilized by aquaculture, which has led to a shift to $n-6$ PUFA-based vegetable (such as soybean and rapeseed) oil products, decreasing the nutritional quality of the farmed fish [8-11]. Furthermore, there is a growing need for dietary n-3 PUFAs and n-3 LC-PUFAs in terrestrial livestock to enrich levels in meat, milk and egg products $[12,13]$.

Potential solutions to the growing demand for $\mathrm{n}-3$ 18C-PUFAs and LC-PUFAs are plant and algae-based sources produced through solar energy-dependent processes [7]. Most plant-sourced, n-3 PUFA-containing oils, such as flaxseed oil, are enriched with the 18C-PUFA $\alpha$-linolenic acid (ALA, 18:4, n-3), which has the potential to be converted to n-3 LC-PUFAs such as eicosapentaenoic acid (EPA, 20:5) and docosahexaenoic acid (DHA, 22:6). However, humans and most animals, including cold-water species of fish, are inefficient at converting ALA into EPA and DHA. The rate-limiting steps in this conversion are the desaturation steps, and particularly the $\Delta 6$ desaturase (Fig. 1). However, the product of $\Delta 6$ desaturase, stearidonic acid (SDA, 18:4, n-3), bypasses this rate-limiting step; several human and animal studies show that seed oils containing SDA are more efficiently converted to EPA than those with ALA [14-17]. SDAcontaining seed oils from relatively rare plant species have been commercialized and common plant seed oils

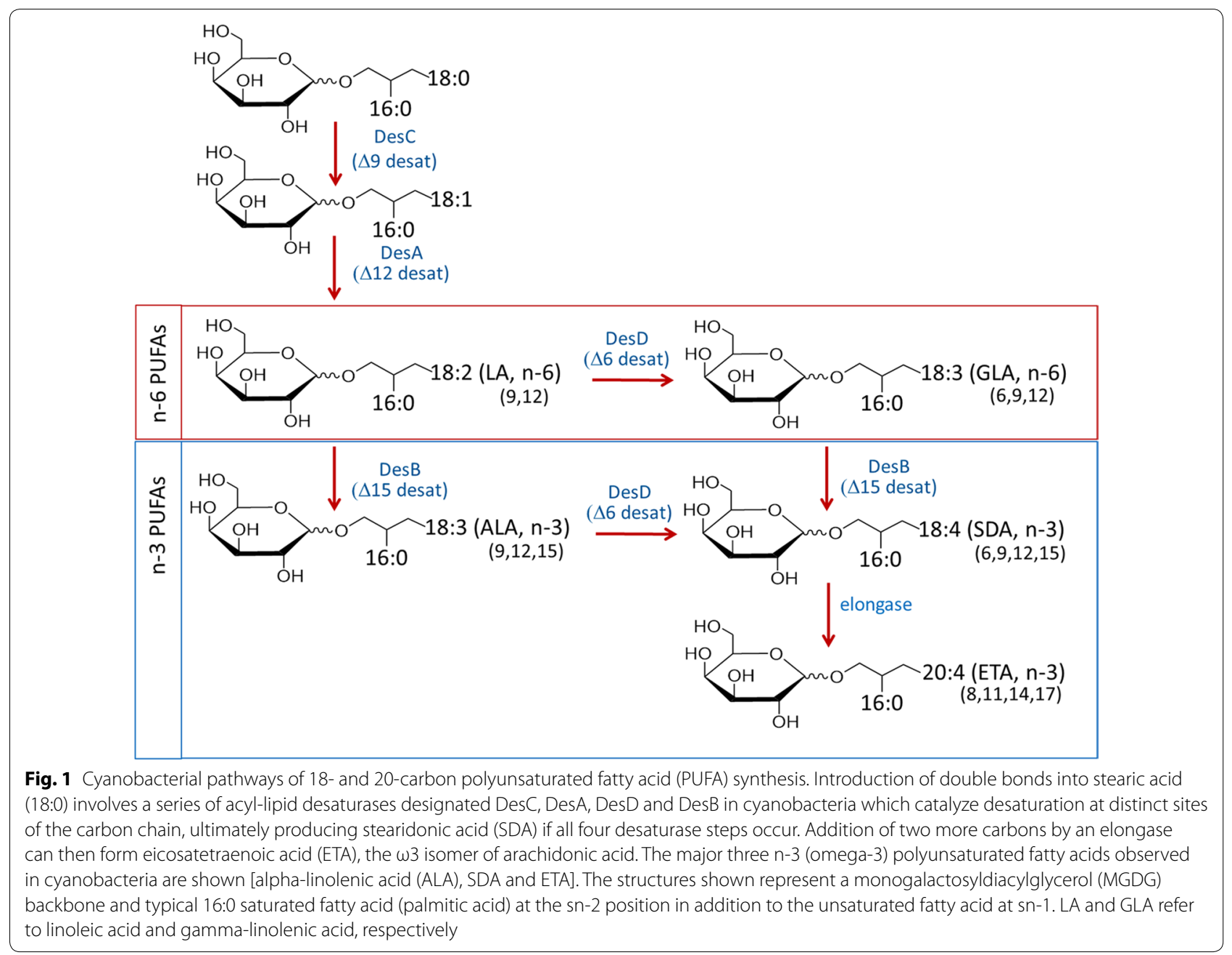


such as soybeans and canola have been genetically engineered to have enriched SDA content $(20-29 \%$ of total fatty acids) $[18,19]$. Human clinical studies show that SDA-enhanced soybean oil significantly elevates n-3 LCPUFAs and improves markers of cardiovascular health $[20,21]$. However, the feasibility of these commercial applications and the stability of these transgenic plants remain to be determined. More recently, there has been a marked increase in the production and sales in human consumer markets of n-3 LC-PUFAs (EPA and DHA) from phototrophic algae. Nevertheless, there are significant production cost barriers in supplying plant and animal sources of $n-3$ PUFAs and LC-PUFAs to the rapidly expanding aquaculture feed and livestock markets.

Cyanobacteria, which can be grown in large quantities requiring only sun, water and trace nutrients, have been subjected to mutagenesis and metabolic engineering for decades in pursuit of sustainable sources for numerous high-value products [22, 23]. Genetic engineering of cyanobacteria through pathway modulation (both interruption or bolstering of existing pathways and introduction of new pathways) is enabling production of energy-containing molecules for use as biofuels [23-25]. Efforts to augment lipid production through improved cyanobacterial hosts and pathway engineering have also been ongoing, but have met with challenges including low yields when the fatty acids produced are secreted into the media as free acids [26-28]. In fact, PUFAs are naturally produced by cyanobacteria as essential constituents of the polar glycolipids in membranes, with the degree of unsaturation of membrane lipids controlling membrane fluidity $[29,30]$. The content of specific PUFAs varies among different cyanobacteria depending on the identity of the desaturase genes present in the genomes. Recent studies have suggested that these glycolipid-associated n-3 PUFAs are more bioavailable than fish and seed-based sources [31]. Due to differences in digestive routes and physical forms (polar vs non-polar lipids) of n-3 PUFA- and LC-PUFA-containing complex lipids, the bioavailability of diverse forms varies considerably. To date, most n-3 PUFAs or LC-PUFAs have been provided to humans and animals complexed to non-polar triglycerides from seed oils or marine fats. However, there are numerous problems with highly enriched triglyceride formulations including the fact that large quantities of such concentrates are typically needed to achieve effective circulating and tissue (especially brain) levels of PUFAs and LC-PUFAs [32]. To overcome these obstacles, ethyl esters, free fatty acids, re-esterified triglycerides or phospholipids (in the case of krill oil) have been formulated, although with varying degrees of success. Cyanobacteria (and dark green plants with abundant chloroplasts) have thylakoid membranes that contain large quantities of the galactose-containing glycolipids monogalactosyldiacylglycerol (MGDG) and digalactosyldiacylglycerol (DGDG) $[33,34]$. Importantly, there is a selective pancreatic lipase (PLRP2) that mobilizes fatty acids from MGDG and DGDG [35], and initial rodent and human studies suggest that ingestion of LC-PUFAs with or complexed to these glycolipids improves their bioavailability $[31,36]$.

As the prokaryotic precursors of chloroplasts, cyanobacteria are biologically simpler than plants and algae, and genetic manipulation is generally more feasible, enabling metabolic reprogramming by engineering as noted above. Importantly for the purpose of this work, they also contain acyl-lipid desaturases and "Group 4" cyanobacteria have the critical four desaturases (DesC, DesA, DesB and DesD, Fig. 1) necessary to convert stearic acid $(18: 0)$ to SDA [37]. However, as opposed to acyl-CoA or acyl-ACP desaturases, these desaturases act directly on fatty acids within the glycolipids, and these lipids account for $\sim 80 \%$ of total lipids in thylakoid membranes [38]. With these understandings, the overall objective of the current study was to engineer cyanobacterial strains to augment the expression of three genes, $\operatorname{des} B$ and $\operatorname{des} D$ encoding acyl-lipid desaturases (known as $\Delta 15$ and $\Delta 6$ desaturases, respectively), and vipp 1 encoding a thylakoid membrane-enhancing protein. The key question this work addresses is whether it is possible to markedly increase the capacity of cyanobacteria to produce SDA and eicosatetraenoic acid (ETA, 20:4, n-3) complexed to highly bioavailable MGDG and DGDG molecular species.

\section{Results}

Generation of plasmids and engineered cyanobacteria, and analyses of total fatty acid content

With the goal of maximizing cyanobacterial omega-3 production focused particularly on SDA, we selected three genes for overexpression which occur naturally in Group 3 and 4 cyanobacteria [37]. Unlike the first two groups, which express just the $\Delta 9$ desaturase (Group 1), or both the $\Delta 9$ and $\Delta 12$ desaturases (DesC and DesA, respectively, Group 2), Group 3 and 4 cyanobacteria also express the $\Delta 6$ and/or $\Delta 15$ desaturases that enable production of the trienoic fatty acids GLA and ALA, and the tetraenoic fatty acid SDA (in the case of Group 4 cyanobacteria which have all four desaturases) [37]. Specificities of the individual desaturases for the sites of double bond insertion in model cyanobacteria have been well established [39] and sequence signatures have emerged to facilitate functional assignment of new sequences [40, 41]. While the $\Delta 6$ desaturase (DesD) acts on LA or ALA to generate GLA or SDA, respectively, the $\Delta 15$ desaturase (DesB) inserts a double bond three carbons from the methyl end, yielding omega-3 products ALA and SDA 
from LA and GLA, respectively [34, 42]. We hypothesized that DesB and DesD overexpressed from a plasmid would impart or augment SDA synthesis in most cyanobacteria (i.e., those of Groups 2 through 4 ).

Previous studies reveal that these desaturase reactions occur within thylakoid membranes, and a thylakoid membrane formation enhancer gene, vipp1 (which encodes vesicle-inducing protein in plastids or Vipp1, also known as IM30) $[38,43]$, was the third protein selected for overexpression to potentially boost levels of newly synthesized PUFAs formed by the enhanced desaturase system. All three synthetic genes $(\operatorname{des} B, \operatorname{des} D$ and vipp1) encoding the authentic cyanobacterial protein products were incorporated into the expression plasmid pAM4418 (Fig. 2a) first described by Taton et al. [45], either singly or in combination with one or two other genes. The constructs were conjugated into Leptolyngbya sp. strain BL0902 (hereafter designated BL0902), a freshwater, filamentous cyanobacterium noted for its excellent growth characteristics and high lipid and especially LA content $[44,45]$. No obvious deleterious effects on growth were observed in any of the seven exconjugants.

All plasmid-bearing cells of BL0902 showed a marked elevation in both saturated and polyunsaturated fatty acids (with monounsaturated levels varying) compared with the wild type (Fig. 2b); the total fatty acid content of the seven exconjugants ranged from 2- to 2.5-fold greater after conjugation. The three-gene-containing plasmid pDBV (Fig. 2a) increased the total fatty acid content from 13 to about $40 \mathrm{mg} / \mathrm{g}$ dry weight and total PUFAs from 5.8 to $16.9 \mathrm{mg} / \mathrm{g}$ dry weight (Fig. $2 \mathrm{~b}$ and Table 1A). Addition of vipp 1 to all desaturase-expressing exconjugants elevated the total fatty acid content (Fig. 2b). To investigate the potential for the pDBV plasmid to impact SDA production in other cyanobacteria, we selected two strains for conjugation with our constructs, Synechococcus sp. PCC7002 and Anabaena sp. PCC7120 (subsequently referred to as 7002 and 7120). Synechococcus elongatus PCC7942 was considered for engineering, but not further studied when initial fatty acid profiling indicated a lack of LA (Additional file 2). Unlike BL0902, strain 7002 engineered with pDBV showed no increase in total lipids while 7120 exhibited a modest increase of $23 \%$ (Additional file 1: Fig. S1).

\section{Modulation of individual fatty acids in engineered strains}

Individual fatty acids (expressed as $\mathrm{mg} / \mathrm{g}$ dry weight or percentage of total fatty acids) from the total lipid extracts of wild type and exconjugants of the three cyanobacterial strains were analyzed by gas chromatography-flame ionization detection (GC-FID) and mass levels compared (Additional files 2, 3). Figure 3a illustrates the effect of inclusion of just one of the three genes

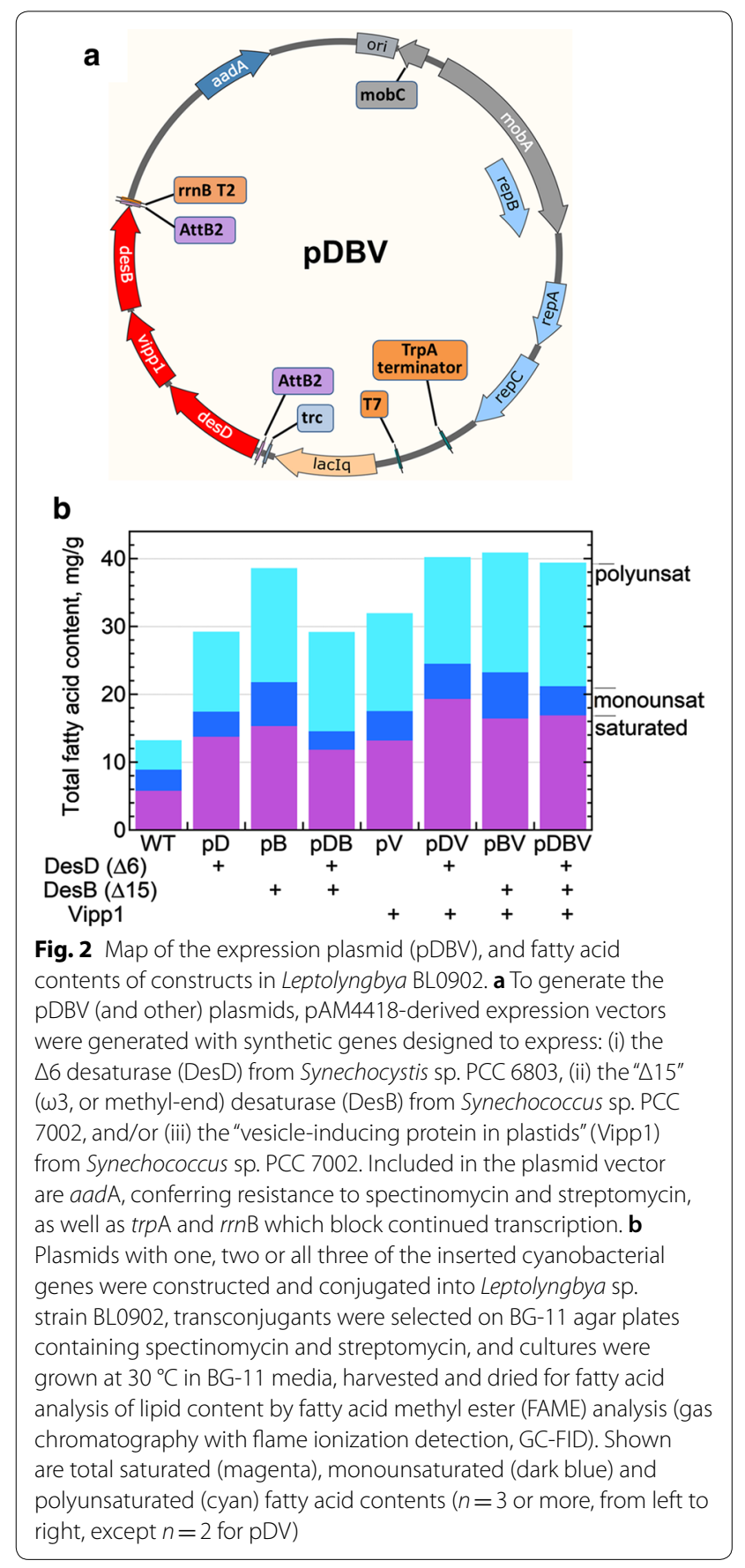

on n-6 and n-3 PUFAs in BL0902, and Fig. 3b summarizes the specific fatty acid contents when two or all three genes are included on the plasmid. Further information comparing the change in mass of pDBV-associated PUFAs with those from the BL0902 strains with the twogene plasmids is also provided in the supplementary data (Additional file 1: Fig. S2). 
Table 1 Summary of pBV and pDBV plasmid effects on cyanobacterial fatty acids

\begin{tabular}{|c|c|c|c|c|}
\hline Host strain & Fatty acid parameter & Wild type (WT) & $\mathrm{WT}+\mathrm{pBV}$ & $\mathrm{WT}+\mathrm{pDBV}$ \\
\hline \multicolumn{5}{|c|}{ Part A. Total fatty acid contents (mg/g dry wt) ${ }^{a}$} \\
\hline \multirow[t]{4}{*}{ Leptolyngbya sp. BL0902 } & Total fatty acids (FAs) & $13.2 \pm 7.6$ & $40.9 \pm 3.0$ & $39.4 \pm 1.8$ \\
\hline & Saturated FAs & $4.3 \pm 2.9$ & $17.6 \pm 1.7$ & $18.2 \pm 0.3$ \\
\hline & Monounsaturated FAs & $3.1 \pm 1.9$ & $6.8 \pm 0.5$ & $4.3 \pm 0.4$ \\
\hline & Polyunsaturated FAs & $5.8 \pm 2.9$ & $16.4 \pm 0.8$ & $16.9 \pm 1.4$ \\
\hline \multicolumn{5}{|c|}{ Part B. $18 \mathrm{C}$ and $20 \mathrm{C}$ omega-3 fatty acid contents (mol\% of total fatty acids) } \\
\hline \multirow[t]{3}{*}{ Leptolyngbya sp. BL0902 } & Total SDA & 0 & 0 & $26.6 \pm 1.0 \%$ \\
\hline & Total SDA + ALA +ETA & $23.8 \pm 1.6 \%$ & $39.0 \pm 1.8 \%$ & $40.1 \pm 3.2 \%$ \\
\hline & Ratio omega-3/omega-6 & 1.2 & 57 & 69 \\
\hline \multirow[t]{3}{*}{ Synechococcus sp. PCC7002 } & Total SDA & 0 & & $17.3 \pm 2.1 \%$ \\
\hline & Total SDA + ALA + ETA & $7.1 \pm 2.0 \%$ & & $40.3 \pm 1.6 \%$ \\
\hline & Ratio omega-3/omega-6 & 0.2 & & 16 \\
\hline
\end{tabular}

No entry means that there is no information for that system

a Measured by gas chromatography and flame ionization of methyl ester-derivatized fatty acyl groups (FAME analysis), reported as mean \pm standard deviation; $n=3$ for all samples except $n=5$ for wild-type Leptolyngbya

In the initial studies with wild-type BL0902, we observed that only LA and ALA were present, implying that DesB (the $\Delta 15$ desaturase) is present and expressed to some extent (in addition to DesA and DesC), but the des $\mathrm{D}$ gene is likely missing (i.e., Leptolyngbya sp. strain BL0902 is likely a member of group 3 $\alpha$ ) [37]. When only des $\mathrm{D}$ (encoding the $\Delta 6$ desaturase) is present on the plasmid, the organism now produces some amount of all five $18 \mathrm{C}$ and $20 \mathrm{C}$ PUFAs, with GLA predominating (consistent with the conversion of LA to GLA catalyzed by DesD). Maximal SDA is seen when both desaturases are included on the plasmid (Fig. 3b), particularly when vipp 1 is also included (SDA as a percent of total FAs is not much changed between $\mathrm{pDB}$ and $\mathrm{pDBV}$, but the total mass increases significantly with the latter, Additional file 1: Fig. S2). With no desD included, expression of des $\mathrm{B}$ provided by the plasmid has a strong effect and leads to considerable accumulation of ALA at the expense of LA in BL0902 with constructs $\mathrm{pB}$ and pBV (gold in Fig. 3a and dark blue in Fig. 3b, Table 1) consistent with the $\Delta 15$ desaturase activity of DesB (Fig. 1). Importantly, the full three-gene plasmid shifted the profile to the greatest quantities of SDA and ETA, with some ALA remaining but no LA or GLA (red in Fig. 3b and c). Total SDA contents reached $26.6 \pm 1.0 \mathrm{~mol} \%$ of total fatty acids in the pDBV exconjugant, and the total n-3 PUFAs including LC-PUFAs (ALA, SDA and ETA) reached $40 \%$ of the lipid content in BL0902 (Table 1). The remarkable shift from n-6 to n-3 PUFA-containing lipids in the pDBV exconjugant was limited from producing additional SDA and ETA primarily by the unavailability of precursor substrates, LA and GLA. With such a depletion of LA and GLA, the pDBV exconjugant produced a ratio of n-3 to n-6 PUFAs of 69:1 (Table 1).

The two other strains of cyanobacteria (7002 and 7120) were tested with and without pDBV and like BL0902, wild-type strains contained no detectable SDA or ETA, but both were produced upon addition of pDBV (Fig. 3c). Quantities of SDA produced by 7002 and 7120 were $31-35 \%$ of that generated by the engineered BL0902 strain (Fig. 3c), an organism which was noted for its favorable lipid composition when first isolated [44]. Although not depicted in Fig. 3, small amounts of the n-3 LC-PUFAs EPA (20:5, n-3) and docosapentaenoic acid (DPA; 22:5, n-3) were also observed in some of the engineered (but not wild type) strains (Additional files $2,3)$. Mass quantities of $0.03 \pm 0.02$ and $0.19 \pm 0.05 \mathrm{mg} / \mathrm{g}$ dry weight were obtained for EPA in engineered 7002 and BL0902, respectively, while DPA was observed at $0.25 \mathrm{mg} / \mathrm{g}$ dry weight in only 7120 (Additional file 1: Fig. $\mathrm{S} 1)$.

\section{Lipidomics analysis}

Lipidomics analysis was performed by liquid chromatography with tandem mass spectrometry (LC-MS/MS) using a high-resolution Q Exactive HF Hybrid Quadrupole-Orbitrap mass spectrometer and was utilized to determine the molecular classes and species of all lipids, but especially those containing ALA, SDA and ETA. More than 300 lipid molecular species were identified in wild-type BL0902 (309 total) and 258 species in the pDBV exconjugants ( $n=4$ per group). Figure 4 illustrates that there are 159 lipid molecular species shared by the wild type and pDBV exconjugants, while 99 lipid 

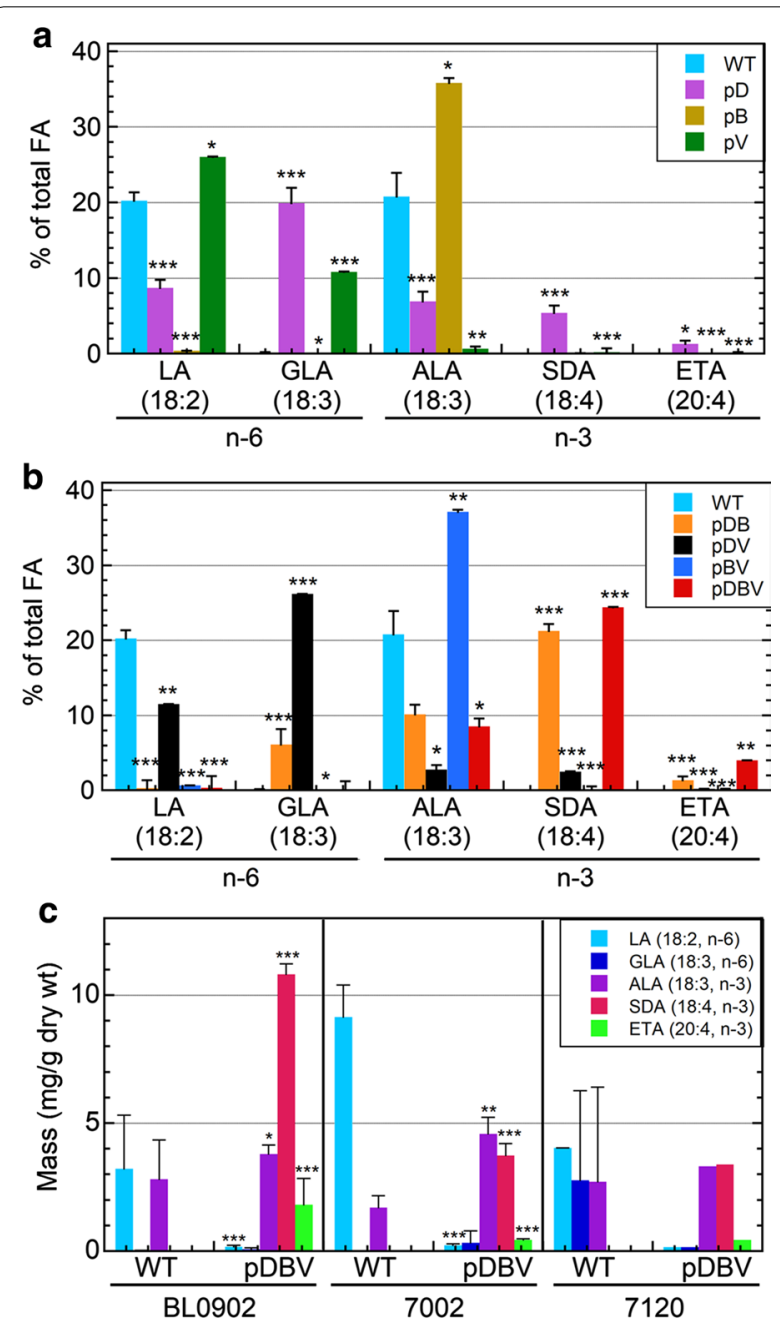

Fig. 3 Quantitative analysis showing 18- and 20-carbon polyunsaturated fatty acids in wild type and engineered cyanobacteria. a, b PUFA analyses of plasmid-bearing Leptolyngbya sp. strain BL0902 as described in Fig. 2b are shown as mean \pm standard deviation for wild type (WT) and single-gene constructs (a), or double and triple-gene constructs (b), expressed as the mol percent of total fatty acids (very similar to weight percent values). (C) PUFAs produced by WT and pDBV-bearing species of cyanobacteria, including Leptolyngbya sp. strain BL0902, Synechococcus sp. PCC 7002 and Anabaena sp. PCC 7120, are shown as averages of the mass ( $\mathrm{mg}$ per $\mathrm{g}$ of dry weight) \pm standard deviation ( $n=3$ or more, from left to right, except $n=2$ and 1 for the 7120 samples). a-c Those exhibiting a statistically significant difference in content compared with WT by a Student's $t$ test are indicated with asterisks $\left({ }^{*} p<0.05,{ }^{* *} p<0.01\right.$, ${ }^{* * *} p<0.001$ )

molecular species were unique to the exconjugants. These unique lipids included ALA-, SDA-, ETA-containing molecular species of MGDG and DGDG classes (Fig. 4b, c). Importantly, SDA is found almost entirely in the MGDG and DGDG species (Table 2, Fig. 4b, c). In contrast, ALA is predominantly in MGDG and DGDG in
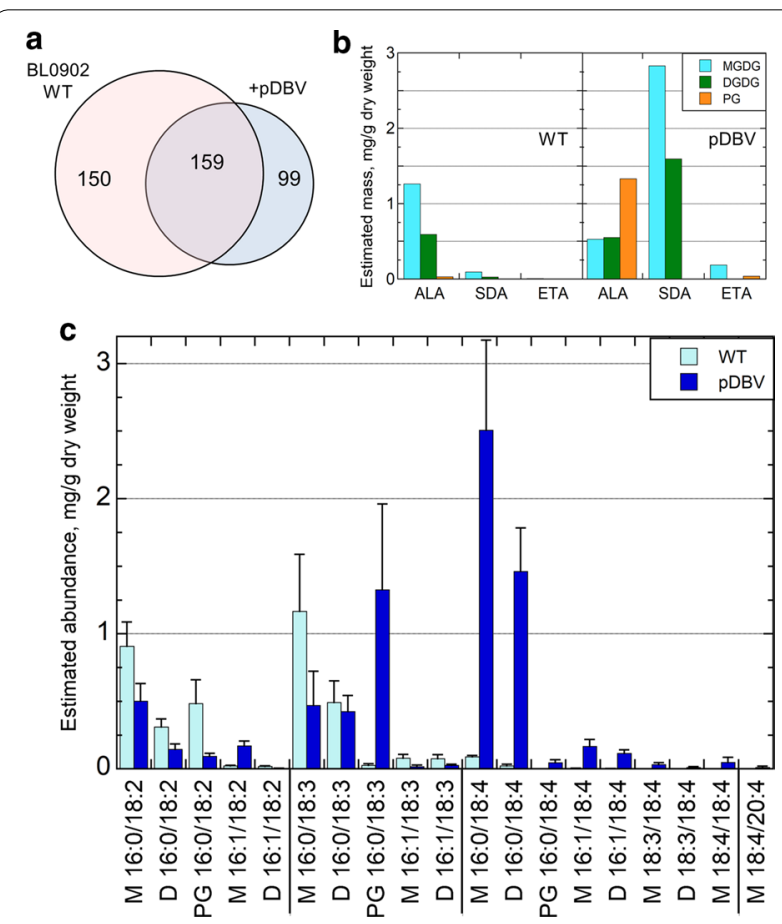

Fig. 4 Lipid molecular species by LC-MS/MS of PUFA distribution in engineered and wild-type Leptolyngbya BL0902. LC-MS/MS was conducted on lipid extracts dissolved in isopropyl alcohol/ methanol (50:50), chromatographed on an Accucore C30 column, and introduced by heated electrospray ionization into a Q Exactive HF Hybrid Quadrupole-Orbitrap Mass Spectrometer with MS scans collected in data-dependent mode, as described in Methods. a Venn diagram of the number of distinct molecular species observed for Leptolyngbya BL0902 without (WT) or with conjugation with pDBV ( $n=4$ for each). b, c 18- and 20-carbon PUFAs were observed to be complexed to monogalactosyldiacylglycerol (MGDG), digalactosyldiacylglycerol (DGDG), or phosphatidylglycerol (PG). (B) Distribution of ALA $(18: 3, n-3)$, SDA $(18: 4, n-3)$ and ETA $(20: 4, n-3)$ among the three glycolipids is shown, illustrating the selectivity for the lipid backbone for each PUFA. c Shown in light blue (WT) and dark blue (engineered with $\mathrm{pDBV}$ ) are the mean \pm standard deviation of estimates of $\mathrm{mg} / \mathrm{g}$ of fatty acids based on (i) normalized peak areas from LC/MS, (ii) fraction of total peak area for each species in a sample, and (iii) known total fatty acid yield for that organism from GC-FID analysis. This treatment assumes that all species exhibit the same ionization efficiency. Species across the bottom refer to the two acyl chains associated with MGDG (M), DGDG (D) or PG. Note the shift from fewer to more double bonds upon introduction of the pDBV plasmid

WT, but is highly enriched in phosphatidylglycerol (PG, $56 \%$ ) in the pDBV exconjugant. ETA is found predominantly in MGDG (84\%) with none detected in DGDG (Fig. 4b).

The individual molecular species of MGDG, DGDG and PG are illustrated in Fig. 4c. Glycolipids contain 18C acyl groups such as 18:0, 18:1 (n-9), 18:2 (n-6), 18:3 (n-3 or $n-6)$ at the sn-1 position and C16 acyl groups including 
Table 2 Lipidomics analysis of molecular species in wild type (WT) and engineered (+pDBV) Leptolyngbya BL0902

\begin{tabular}{|c|c|c|c|c|c|c|c|c|}
\hline \multirow[t]{2}{*}{ Molecular species ${ }^{a}$} & \multicolumn{2}{|l|}{ MGDG } & \multicolumn{2}{|l|}{ DGDG } & \multicolumn{2}{|l|}{ SQDG } & \multicolumn{2}{|l|}{ PG } \\
\hline & WT & $+p D B V$ & WT & $+\mathrm{pDBV}$ & WT & $+\mathrm{pDBV}$ & WT & $+p D B V$ \\
\hline $14: 0 / 16: 0$ & & & $0.005 \pm 0.001$ & $0.002 \pm 0.001$ & & & $0.001 \pm 0.001$ & $0.000 \pm 0.000$ \\
\hline $14: 0 / 18: 3$ & & & $0.013 \pm 0.001$ & $0.010 \pm 0.003$ & & & & \\
\hline 14:0/18:4 & $\underline{0.000 \pm 0.000}$ & $\underline{0.009 \pm 0.002}$ & $\underline{0.000 \pm 0.000}$ & $\underline{0.006 \pm 0.002}$ & & & & \\
\hline 16:0/16:0 & $0.066 \pm 0.024$ & $0.129 \pm 0.038$ & $0.043 \pm 0.022$ & $0.150 \pm 0.111$ & $0.008 \pm 0.005$ & $0.016 \pm 0.015$ & $0.037 \pm 0.023$ & $0.113 \pm 0.037$ \\
\hline $16: 0 / 16: 1$ & $0.161 \pm 0.028$ & $0.201 \pm 0.080$ & $0.045 \pm 0.018$ & $0.078 \pm 0.024$ & & & $0.077 \pm 0.025$ & $0.089 \pm 0.028$ \\
\hline 16:0/16:2 & $0.021 \pm 0.006$ & $0.016 \pm 0.005$ & $0.011 \pm 0.004$ & $0.006 \pm 0.001$ & & & $0.004 \pm 0.002$ & $0.007 \pm 0.003$ \\
\hline $16: 0 / 16: 3$ & $0.001 \pm 0.000$ & $0.040 \pm 0.018$ & & & & & & \\
\hline $16: 0 / 16: 4$ & $0.000 \pm 0.000$ & $0.010 \pm 0.003$ & & & & & & \\
\hline $16: 0 / 17: 0$ & $0.002 \pm 0.001$ & $0.007 \pm 0.002$ & $0.002 \pm 0.002$ & $0.012 \pm 0.005$ & & & $0.000 \pm 0.001$ & $0.001 \pm 0.002$ \\
\hline $16: 0 / 17: 1$ & $0.040 \pm 0.010$ & $0.066 \pm 0.020$ & $0.009 \pm 0.004$ & $0.016 \pm 0.003$ & & & $0.013 \pm 0.005$ & $0.044 \pm 0.020$ \\
\hline $16: 0 / 17: 2$ & $0.028 \pm 0.006$ & $0.034 \pm 0.012$ & $0.011 \pm 0.003$ & $0.009 \pm 0.002$ & & & $0.005 \pm 0.002$ & $0.001 \pm 0.002$ \\
\hline 16:0/17:3 & $0.020 \pm 0.008$ & $0.024 \pm 0.004$ & $0.010 \pm 0.006$ & $0.010 \pm 0.006$ & & & $0.000 \pm 0.000$ & $0.014 \pm 0.005$ \\
\hline $16: 0 / 17: 4$ & $0.000 \pm 0.000$ & $0.045 \pm 0.014$ & $0.000 \pm 0.000$ & $0.015 \pm 0.003$ & & & & \\
\hline $16: 0 / 18: 0$ & $0.008 \pm 0.005$ & $0.020 \pm 0.019$ & $0.007 \pm 0.008$ & $0.029 \pm 0.011$ & & & $0.002 \pm 0.002$ & $0.006 \pm 0.002$ \\
\hline $16: 0 / 18: 1$ & $0.410 \pm 0.088$ & $0.488 \pm 0.180$ & $0.087 \pm 0.044$ & $0.185 \pm 0.057$ & & & $0.275 \pm 0.068$ & $0.397 \pm 0.176$ \\
\hline $16: 0 / 18: 2$ & $0.908 \pm 0.179$ & $0.501 \pm 0.132$ & $0.311 \pm 0.059$ & $0.145 \pm 0.039$ & & & $0.484 \pm 0.177$ & $0.093 \pm 0.022$ \\
\hline 16:0/18:3 & $1.166 \pm 0.421$ & $0.469 \pm 0.254$ & $0.491 \pm 0.160$ & $0.424 \pm 0.118$ & & & $0.027 \pm 0.013$ & $1.325 \pm 0.636$ \\
\hline$\underline{16: 0 / 18: 4}$ & $\underline{0.090 \pm 0.011}$ & $\underline{2.507 \pm 0.665}$ & $\underline{0.022 \pm 0.013}$ & $\underline{1.462 \pm 0.321}$ & & & $\underline{0.001 \pm 0.001}$ & $\underline{0.046 \pm 0.021}$ \\
\hline 16:0/19:1 & $0.007 \pm 0.001$ & $0.017 \pm 0.006$ & $0.002 \pm 0.001$ & $0.004 \pm 0.001$ & & & & \\
\hline $16: 0 / 19: 2$ & $0.006 \pm 0.004$ & $0.002 \pm 0.001$ & $0.003 \pm 0.002$ & $0.001 \pm 0.000$ & & & & \\
\hline $16: 0 / 19: 3$ & $0.002 \pm 0.001$ & $0.007 \pm 0.001$ & & & & & & \\
\hline $16: 0 / 20: 0$ & $0.000 \pm 0.000$ & $0.001 \pm 0.001$ & & & & & & \\
\hline $16: 0 / 20: 2$ & $0.004 \pm 0.003$ & $0.003 \pm 0.001$ & $0.003 \pm 0.003$ & $0.001 \pm 0.000$ & & & & \\
\hline 16:0/20:3 & $0.012 \pm 0.009$ & $0.033 \pm 0.010$ & $0.005 \pm 0.004$ & $0.010 \pm 0.003$ & & & $0.001 \pm 0.002$ & $0.004 \pm 0.001$ \\
\hline$\underline{16: 0 / 20: 4}$ & $\underline{0.006 \pm 0.004}$ & $\underline{0.174 \pm 0.065}$ & & & & & $\underline{0.000 \pm 0.000}$ & $\underline{0.037 \pm 0.021}$ \\
\hline$\underline{16: 0 / 20: 5}$ & $\underline{0.077 \pm 0.010}$ & $\underline{0.122 \pm 0.070}$ & & & & & & \\
\hline $16: 1 / 16: 1$ & $0.005 \pm 0.001$ & $0.007 \pm 0.002$ & $0.005 \pm 0.001$ & $0.003 \pm 0.001$ & & & & \\
\hline $16: 1 / 16: 2$ & $0.001 \pm 0.001$ & $0.000 \pm 0.000$ & & & & & & \\
\hline $16: 1 / 17: 3$ & $0.001 \pm 0.001$ & $0.001 \pm 0.000$ & $0.001 \pm 0.001$ & $0.000 \pm 0.000$ & & & & \\
\hline $16: 1 / 18: 2$ & $0.022 \pm 0.004$ & $0.171 \pm 0.036$ & $0.019 \pm 0.005$ & $0.004 \pm 0.001$ & & & & \\
\hline $16: 1 / 18: 3$ & $0.080 \pm 0.028$ & $0.018 \pm 0.011$ & $0.076 \pm 0.030$ & $0.026 \pm 0.009$ & & & $0.000 \pm 0.000$ & $0.006 \pm 0.001$ \\
\hline$\underline{16: 1 / 18: 4}$ & $\underline{0.003 \pm 0.001}$ & $\underline{0.166 \pm 0.051}$ & $\underline{0.002 \pm 0.001}$ & $\underline{0.115 \pm 0.027}$ & & & & \\
\hline 17:0/18:3 & $0.001 \pm 0.000$ & $0.002 \pm 0.001$ & & & & & & \\
\hline$\underline{17: 0 / 18: 4}$ & $\underline{0.001 \pm 0.000}$ & $\underline{0.006 \pm 0.004}$ & & & & & & \\
\hline $17: 1 / 18: 3$ & $0.001 \pm 0.001$ & $0.001 \pm 0.001$ & $0.001 \pm 0.001$ & $0.000 \pm 0.000$ & & & & \\
\hline $17: 5 / 23: 2$ & & & & & $1.089 \pm 0.123$ & $2.172 \pm 0.449$ & & \\
\hline $17: 5 / 23: 3$ & & & & & $0.005 \pm 0.003$ & $0.006 \pm 0.003$ & & \\
\hline 18:0/18:1 & $0.001 \pm 0.001$ & $0.002 \pm 0.001$ & & & & & $0.001 \pm 0.000$ & $0.002 \pm 0.001$ \\
\hline $18: 1 / 18: 2$ & $0.005 \pm 0.005$ & $0.001 \pm 0.001$ & & & & & $0.001 \pm 0.000$ & $0.008 \pm 0.003$ \\
\hline $18: 1 / 18: 3$ & & & $0.002 \pm 0.001$ & $0.071 \pm 0.027$ & & & & \\
\hline 18:2/18:2 & $0.009 \pm 0.003$ & $0.011 \pm 0.004$ & $0.003 \pm 0.002$ & $0.001 \pm 0.001$ & & & & \\
\hline $18: 2 / 18: 3$ & $0.005 \pm 0.004$ & $0.005 \pm 0.004$ & $0.005 \pm 0.003$ & $0.006 \pm 0.005$ & & & & \\
\hline 18:3/18:3 & $0.003 \pm 0.003$ & $0.001 \pm 0.001$ & $0.002 \pm 0.001$ & $0.000 \pm 0.000$ & & & & \\
\hline$\underline{18: 3 / 18: 4}$ & $\underline{0.000 \pm 0.000}$ & $\underline{0.032 \pm 0.015}$ & $\underline{0.000 \pm 0.000}$ & $\underline{0.012 \pm 0.004}$ & & & & \\
\hline$\underline{18: 4 / 18: 4}$ & $\underline{0.000 \pm 0.000}$ & $\underline{0.049 \pm 0.037}$ & & & & & & \\
\hline $18: 4 / 20: 4$ & $\underline{0.000 \pm 0.000}$ & $\underline{0.012 \pm 0.009}$ & & & & & & \\
\hline
\end{tabular}

Values reported are estimates of $\mathrm{mg} / \mathrm{g}$ of total fatty acid based on (i) normalized peak areas from LC/MS, (ii) fraction of total peak area for each species in a sample, and (iii) known total fatty acid yield for that organism from GC-FID analysis. This treatment assumes that all species exhibit the same ionization efficiency. Shown are mean \pm standard deviation for wild-type (WT) Leptolyngbya BL0902 and the same strain with pDBV ( $n=4$ for each). Molecular species containing at least one acyl group with SDA (18:4), ETA (20:4) or EPA (20:5) are underlined. No entry means that the species was not observed in the WT or pDBV samples in that category 
Table 2 (continued)

MGDG monogalactosyldiacylglycerol, DGDG digalactosyldiacylglycerol, SQDG sulfoquinovosyldiacylglycerol, $P G$ phosphatidylglycerol

a Molecular species shown are for the two fatty acyl chains, giving carbon chain length and number of double bonds for each (e.g., 16:0 means 16 carbons long with 0 double bonds)

$16: 0,16: 1,16: 2,16: 3$ at the sn-2 position of the molecule [34]. In the case of MGDG, DGDG, and PG containing ALA, SDA or ETA, the most common fatty acid observed at the sn-2 acyl position is palmitic acid (16:0) (89-95\% of the time, Additional file 1: Fig. S3). Interestingly, the pDBV exconjugants produced several novel and unexpected MGDG and DGDG molecular species containing $18 \mathrm{C}$ and/or 20C PUFAs in both (sn-1 and sn-2) positions, including SDA and ALA, SDA and SDA, or SDA and ETA (Fig. 4c).

A second experiment comparing BL0902 and 7002 samples with and without pDBV demonstrated similar molecular profiles, with ALA/ALA, ALA/SDA, ALA/ETA, SDA/SDA and SDA/ETA "double omega-3" chains among the MGDG and DGDG molecular species, as well as the SDA/SDA molecular species in sulfoquinovosyldiacylglycerol (SQDG). In fact, the main difference between the outputs from the two experiments was an accumulation of SDA and especially ALA in SQDG rather than PG (Additional file 1: Table S2). There also were other rarer PUFAs induced by $\mathrm{pDBV}$ in both BL0902 and 7002 including 16:3, 16:4, 17:2, 17:4, 20:3, and 20:5 (Additional file 1: Table S1 and Additional file 2). Another highly unusual fatty acid, 18:5, was present in MGDG and SQDG in the second experiment and the SQDG in particular was induced upon addition of pDBV to both BL0902 and 7002.

\section{Discussion}

The primary objective of this work was to determine the capacity of cyanobacteria strains to produce SDA as well as elongation and desaturation metabolites (ETA and EPA, respectively) complexed to potentially more bioavailable glycolipids (MGDG and DGDG). This was accomplished by conjugal transfer into three cyanobacteria of a three-gene plasmid, pDBV, which encodes the thylakoid membrane-promoting protein Vipp1 and two acyl-lipid desaturases ( $\Delta 6$ and $\Delta 15$ desaturases) that occur naturally in cyanobacteria. The total yield of fatty acids increased threefold in the pDBV exconjugants, and SDA levels which were at baseline in the wild-type cyanobacteria rose to $26.6 \mathrm{~mol} \%$ in Leptolyngbya BL0902 and $17.3 \mathrm{~mol} \%$ in Synechococcus PCC 7002. Importantly, n-3 PUFAs and LC-PUFAs (ALA + SDA + ETA) comprised $\sim 40 \%$ of total fatty acids in engineered Leptolyngbya BL0902, and these were incorporated into MGDG and DGDG with a n-3 to n- 6 PUFA ratio of $>50: 1$ (Table 1). In comparison, similar studies reported by
Chen et al. [46] expressing tagged versions of DesB, or both DesB and DesD, in Synechocystis sp. PCC6803 achieved levels of SDA of around $10.8 \%$ and 13.1\%, respectively (less than half as much as we observed with pDBV-engineered Leptolyngbya BL0902) when grown at $20{ }^{\circ} \mathrm{C}$, less if grown at the higher temperature of $30{ }^{\circ} \mathrm{C}$ that we used here (Table 3 ). In terms of total mass accumulated, SDA in our pDBV Leptolyngbya was produced (in about mid-log phase) at $10.8 \pm 0.4 \mathrm{mg} / \mathrm{g}$ dry weight, which is comparable to the maximum observed previously of $12.2 \pm 2.4 \mathrm{mg} / \mathrm{g}$ by Yoshino et al. under high incident light $\left[150 \mu \mathrm{mol}\right.$ photons $/\left(\mathrm{m}^{2} \cdot \mathrm{s}\right)$, compared with 20-30 $\mu \mathrm{mol}$ photons $/\left(\mathrm{m}^{2} \cdot \mathrm{s}\right)$ in our studies] (Table 3 ) [47].

The rationale for the addition of vipp1 to the plasmid was to enhance thylakoid membranes and thus the content of MGDG and DGDG as well as the potential activities of the acyl-lipid desaturases (DesC, DesA, DesB and DesD). While Vipp1 is reported to promote thylakoid membrane biogenesis and maintenance in plants and cyanobacteria associated with enhanced photosynthetic machinery $[48,49]$, the effect of this gene on PUFA and LC-PUFA content has not been previously reported. It is well established that n-3 PUFA contents of cyanobacteria are significantly affected by temperature and light intensity [47, 50-52]. The SDA and ETA contents may therefore be even further enhanced in these cyanobacteria by growth at lower temperatures and higher light intensities and further engineering of the cyanobacteria to enhance their capacity to generate more precursor substrates (LA, GLA or ALA) necessary for SDA and ETA production. For example, Spirulina strains can contain $\sim 50 \%$ of their total fatty acids as LA and GLA [53], and other cyanobacterial strains have been engineered that contain 25-82\% of their total fatty acids as LA and ALA [46, 47, 54].

Given SDA's stability relative to n-3 LC-PUFAs in food matrices and its capacity to be more efficiently (than ALA) converted to health-promoting EPA in humans, fish and livestock, a great deal of effort has gone into finding natural systems and designing new engineered pathways that produce high quantities of SDA and high ratios of n-3 to n-6 PUFAs [55]. SDA is seldom naturally found in cyanobacteria [56] unless at least one acyl-lipid desaturase is provided on a multicopy vector [47], and even then, the maximal content of $26.6 \%$ SDA reached in the current study is as we have noted at least twofold higher than the previous engineered strains (Table 3) $[46,47]$. SDA-producing transgenic soybean oil contains 
Table 3 Content of $18 \mathrm{C}$ omega-3 fatty acids in engineered cyanobacteria from this and previous studies

\begin{tabular}{|c|c|c|c|c|c|c|}
\hline Species & $\begin{array}{l}\text { Vector } \\
\text { for engineering }\end{array}$ & $\operatorname{Temp}\left({ }^{\circ} \mathrm{C}\right)$ & $\begin{array}{l}\text { Photon flux density, } \\
\mu \mathrm{mol} \text { photons }\left(\mathrm{m}^{2} \text {. }\right. \\
\left.\mathrm{s}^{-1}\right)^{-1}\end{array}$ & $\begin{array}{l}\text { ALA }(18: 3, n-3), \\
\text { mol\% of total } \\
\text { FAs }^{\mathrm{a}}\end{array}$ & $\begin{array}{l}\text { SDA }(18: 4, n-3), \\
\text { mol\% of total } \\
F^{a} s^{a}\end{array}$ & References \\
\hline \multirow{3}{*}{$\begin{array}{l}\text { Leptolyngbya sp. strain } \\
\text { BL0902 }\end{array}$} & None (wt) & 30 & $20-30$ & $22.6 \pm 11.5$ & n.d. & \multirow[t]{3}{*}{ This study } \\
\hline & $\begin{array}{l}\text { With pBV (to express } \\
\text { DesB and Vipp1) })^{b}\end{array}$ & 30 & $20-30$ & $37.7 \pm 1.7$ & n.d. & \\
\hline & $\begin{array}{l}\text { With pDBV (to express } \\
\text { DesD, DesB and } \\
\text { Vipp1) }\end{array}$ & 30 & $20-30$ & $9.3 \pm 0.9$ & $26.6 \pm 1.0$ & \\
\hline \multirow{4}{*}{$\begin{array}{l}\text { Synechococcus elonga- } \\
\text { tus PCC } 7942\end{array}$} & None (wt) & 22 & 60 & n.d. & n.r. & \multirow[t]{4}{*}{ Santos-Merino et al. [54] } \\
\hline & \multirow{2}{*}{$\begin{array}{l}\text { MSM26 (strain with } \\
\text { overexpressed desA } \\
\text { and desB) }\end{array}$} & 22 & 60 & $7.6 \pm 1.5$ & n.r. & \\
\hline & & 22 & 250 & $8.8 \pm 1.8$ & n.r. & \\
\hline & 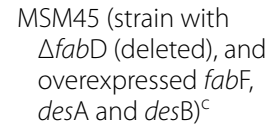 & 22 & 60 & $22.6 \pm 1.5$ & n.r. & \\
\hline \multirow{5}{*}{$\begin{array}{l}\text { Synechococcus sp. } \\
\text { strain NKBG } 15041 \mathrm{c}\end{array}$} & Empty vector & 25 & 50 & $\sim 21 \pm 4^{d}$ & n.d. & \multirow[t]{5}{*}{ Yoshino et al. [47] } \\
\hline & \multirow{4}{*}{$\begin{array}{l}\text { Vector for des } D \\
\text { overexpression }^{\mathrm{e}}\end{array}$} & 25 & 50 & $20.6 \pm 3.7$ & $2.1 \pm 0.5$ & \\
\hline & & 25 & 100 & $45.7 \pm 7.4$ & $5.1 \pm 0.6$ & \\
\hline & & 25 & 150 & $53.1 \pm 1.3$ & $6.2 \pm 0.5$ & \\
\hline & & 30 & 50 & $9.0 \pm 6.3$ & $0.9 \pm 0.4$ & \\
\hline \multirow{3}{*}{$\begin{array}{l}\text { Synechococcus sp. } \\
\text { PCC7002 }\end{array}$} & None (wt) & 30 & 60 & $5.2 \pm 0.3$ & n.d. & \multirow[t]{3}{*}{ Dong et al. [80] } \\
\hline & $\begin{array}{l}\text { Syd15D (for desB } \\
\text { overexpression) }^{f}\end{array}$ & 30 & 60 & $28.3 \pm 2.3$ & n.d. & \\
\hline & $\begin{array}{l}\text { Syd6Dd15D (for } \\
\text { desD and desB } \\
\text { overexpression), fef }\end{array}$ & 30 & 60 & $6.6 \pm 0.7$ & $11.6 \pm 1.6$ & \\
\hline \multirow{6}{*}{$\begin{array}{l}\text { Synechocystis sp. PCC } \\
6803\end{array}$} & \multirow[t]{2}{*}{ None (wt) } & 20 & 40 & $2.5 \pm 0.3$ & $1.5 \pm 0.2$ & \multirow[t]{6}{*}{ Chen et al. [46] } \\
\hline & & 30 & 40 & $1.5 \pm 0.2$ & $1.2 \pm 0.3$ & \\
\hline & \multirow{2}{*}{$\begin{array}{l}\text { Sy15 (for desB } \\
\text { overexpression) }^{g}\end{array}$} & 20 & 40 & $23.1 \pm 2.3$ & $10.8 \pm 1.6$ & \\
\hline & & 30 & 40 & $17.5 \pm 2.3$ & $9.1 \pm 1.3$ & \\
\hline & \multirow{2}{*}{$\begin{array}{l}\text { Sy15Sy6 (for } \\
\text { desB and desD } \\
\text { overexpression) }^{\text {gh h }}\end{array}$} & 20 & 40 & $16.4 \pm 1.9$ & $13.1 \pm 1.3$ & \\
\hline & & 30 & 40 & $23.6 \pm 3.4$ & $7.8 \pm 0.7$ & \\
\hline
\end{tabular}

n.d. not detected, n.r. not reported

a Expressed as mean \pm standard deviation

b pBV encodes Synechococcus sp. PCC7002 DesB for $\triangle 15$ desaturase (also known as $\omega 3$ or methyl end desaturase) and Synechococcus sp. PCC7002 Vipp1 for inducing thylakoid membranes; pDBV is the three-gene plasmid derived from pAM4418 (Fig. 2) developed in this work which includes the two genes of pBV plus Synechocystis sp. PCC6803 desD for $\Delta 6$ desaturase expression

c desA and desB were from Synechococcus sp. PCC7002

d Estimated from mass if total fatty acid content is same as transformed strain

e desD was from Synechocystis sp. PCC6803

f desB was from Synechocystis sp. PCC6803

9 desB was from Synechocystis sp. PCC6803 plus a C-terminal FLAG tag

h desD was from Synechocystis sp. PCC6803 plus a C-terminal His tag

$20-26 \%$ of the total fatty acids as SDA with n-3 to n-6 PUFA ratios of $\sim 1$ or lower $[19,21,55,57]$, and seed oil from Echium plantagineum naturally contains $\sim 13 \%$ of the total fatty acids as SDA $[15,58,59]$.

Lipidomics analysis identified complex lipids and individual molecular species containing newly synthesized n-3 PUFAs and LC-PUFAs. Greater than 99\% of SDA and ETA resided in MGDG and DGDG with the majority at the sn-1 position and palmitic acid at the sn-2 position of the glycolipid backbone (Fig. $4 \mathrm{~b}$ and Additional file 1: Fig. S3). Additionally, several highly unusual MGDG and DGDG molecular species containing SDA at both acyl positions or ALA:SDA, ALA:ETA and SDA:ETA combinations were detected. ETA is a rare $\mathrm{n}-3 \mathrm{LC}$-PUFA in nature comprising 1\% of fish oils. ETA is also found in triglycerides of transgenic seeds 
from Camelina and in New Zealand green-lipped mussel (Perna canaliculus) [60, 61]. ETA (also known as omega-3 arachidonic acid), an elongation product of SDA, is a structural analog of the n-6 arachidonic acid. Previous studies have demonstrated ETA's capacity to serve as a dual inhibitor of cyclooxygenases (COX1 and COX2) and lipoxygenases that can block the production of several classes of pro-inflammatory eicosanoids including leukotrienes, prostaglandins, and thromboxanes $[62,63]$. ETA has also been demonstrated to compete with arachidonic acid at the arachidonoyl-CoA synthetase step thereby preventing arachidonic acid uptake [61, 64]. Importantly, lipid extracts from New Zealand green-lipped mussel also have been shown to have benefits in patients with atopic asthma [65]. Future studies will determine whether molecular species of MGDG and DGDG such as ALA:ETA, SDA:ETA and ETA:ETA have the potential to serve as bioavailable anti-inflammatory compounds.

Significant advantages of sourcing SDA and ETA from cyanobacteria include: (1) they require minimal nutritional demands, relying on photosynthesis and carbon dioxide rather than fermentable sugars, and do not require arable land for production [66]; (2) they are able to serve directly as single ingredient feeds for aquaculture and livestock (simply by drying and being fed as flakes or pellets), therefore offering the possibility of less labor- and land-intensive cultivation of such feeds [22]; (3) cyanobacteria such as Spirulina are currently used as food supplements because of their high protein content and digestibility [22]; and (4) SDA and ETA formed in cyanobacteria, as shown here, are complexed to bioavailable, polar glycolipids. Human and rodent studies show that high doses of echium oil reduce circulating triglycerides $[15,67]$ and that $>1 \mathrm{~g} /$ day of SDA from transgenic soybean oil is effective at raising tissue membrane levels of EPA and improving the omega- 3 index in humans (erythrocyte EPA and DHA) [21, 68-70]. SDA-enriched soybean oil fed to laying hens performs better than ALA at enriching eggs with n-3 LC-PUFAs and particularly EPA [17], and similar effects were obtained with meat from broiler chickens [71]. Echium oil also enhances total n-3 PUFA levels, including EPA, in the milk of dairy cattle [72]. However, there continues to be substantial barriers to the supplementation of SDA complexed to triglycerides (as found in seed and soybean oils), and this has limited the widespread use of SDA; furthermore, initial studies suggest that PUFAs or LC-PUFAs complexed to MGDG and DGDG may provide greater bioavailability than non-polar, triglyceride-containing oils and phospholipids found in krill oil [31].

\section{Conclusions}

Here, we demonstrate that cyanobacteria, and especially Leptolyngbya BL0902, bioengineered with cyanobacterial lipid biosynthetic promoting genes, produce large quantities of SDA up to about $27 \%$ of total fatty acids. Importantly, both newly synthesized SDA and ETA are found conjugated to galactose-containing glycerol backbones in what initial studies indicate is a more bioavailable polar lipid form than the neutral storage lipids like triacylglycerols of oils. Additionally, several novel and potentially beneficial molecular species of MGDG and DGDG are formed in these cyanobacteria that may serve as highly bioavailable, anti-inflammatory compounds. SDA-producing cyanobacteria as developed here are thus promising, sustainable sources of omega-3 PUFAs that could replace unstable fish oil products $[73,74]$ and fish meal as nutritional supplements for human, agricultural and aquacultural use.

\section{Methods \\ Molecular biology approaches}

Overall, the goal of the project was to generate, for testing in various cyanobacteria, a set of plasmids expressing one, two or three cyanobacterial genes that we hypothesized would enable stearidonic acid (SDA) production in a wide range of cyanobacteria (as long as they were already able to produce the dienoic fatty acid linoleic acid, 18:2). The three genes of interest for this work were $\operatorname{des} \mathrm{D}$, des $\mathrm{B}$ and vipp 1 , for which we designed coding sequences based on the corresponding protein sequences from well-documented sources: DesD, the acyl-lipid $\Delta 6$-desaturase, was from Synechocystis sp. PCC 6803; DesB, the acyl-lipid $\omega 3$-desaturase (alternatively named methyl-end or $\Delta 15$ desaturase) was from Synechococcus sp. PCC 7002; and Vipp1, dubbed the "Vesicle-inducing protein in plastids", was from Synechococcus sp. PCC 7002. Each protein sequence was used to design the synthetic gene, codon optimized for expression in E. coli (given a lack of options for cyanobacteria). Each cloned insert obtained from GenScript was then amplified by polymerase chain reaction (PCR) and cloned into the Gateway donor plasmid, pENTR/SD/D-topo (Invitrogen), which provides an upstream Shine-Dalgarno sequence (ribosome-binding site) that is known to function in cyanobacteria. Sequences of all plasmid inserts were verified and transferred into pAM4418 using the Gateway recombination system (Invitrogen) and Invitrogen's LR Clonase II Enzyme Mix, with verification of positive clones by restriction digestion. Details of the cloning steps varied with the construct and are given below. The expression vector used, pAM4418, is a broad host range, E. coli-cyanobacteria shuttle plasmid that confers resistance to streptomycin and spectinomycin and contains 
both the lacI ${ }^{\mathrm{q}}$ repressor and the $\operatorname{trc}$ promoter from E. coli, in addition to the Gateway recombination cassette [44].

Using the overall approaches outlined above, the following seven expression plasmids were generated: (a) pD: encodes DesD, the $\Delta 6$ desaturase; (b) pB: encodes DesB, the $\Delta 15$ desaturase; (c) pDB: encodes both DesD and DesB; (d) pV: encodes Vipp1; (e) pDV: encodes both DesD and Vipp1; (f) pBV: encodes both DesB and Vipp1; and (g) pDBV: encodes all three, DesD, DesB and Vipp1. The contents of these vectors are also summarized at the bottom of Fig. 2b.

In order to create the engineered pENTR plasmids to generate $\mathrm{pD}$ and $\mathrm{pB}, \operatorname{des} \mathrm{D}$ and $\operatorname{des} \mathrm{B}$, respectively, were PCR amplified using primers which added the sequence CACC before the initiating ATG codon and a Xho 1 restriction site following the termination codon, enabling directional cloning of the PCR product into pENTR/ $\mathrm{SD} / \mathrm{D}$-topo. For the next two constructs, pDV and pBV, the downstream $X h o I$ restriction sites after each desaturase-encoding gene, in combination with the AscI site in the $\mathrm{pENTR/SD/D-topo} \mathrm{plasmid,} \mathrm{provided} \mathrm{sites} \mathrm{for} \mathrm{inser-}$ tion of vipp 1 ; the vipp 1 -containing fragment was excised from the Genscript plasmid using XhoI and $A s c 1$, then ligated following each of the two desaturase genes into the $\mathrm{pENTR/SD/D-topo} \mathrm{clones} \mathrm{described} \mathrm{above.} \mathrm{To} \mathrm{cre-}$ ate the pENTR plasmid encoding only Vipp1 (to generate $\mathrm{pV}$ ), vipp 1 from Genscript was PCR amplified as described above for des $\mathrm{D}$ and $\operatorname{des} \mathrm{B}$ to enable directional cloning of the PCR product into $\mathrm{pENTR/SD/D-topo.}$

In order to create $\mathrm{pDBV}$, the $\operatorname{des} B$ sequence from GenScript was amplified by PCR to introduce a HindIII site plus a ribosome-binding site on the $5^{\prime}$ end and an $A s c 1$ restriction site on the $3^{\prime}$ end. The PCR product was digested with HindIII and AscI and ligated into the pENTR/SD/D-topo plasmid already containing desD and vipp1, digested with the same two restriction enzymes.

Finally, the pENTR plasmid used to generate pDB, encoding only DesD and DesB, was derived from the pENTR plasmid containing all three genes (above) by digestion with HindIII and XhoI to remove the Vipp1encoding gene, filling in the ends with dNTPs and DNA polymerase (Klenow fragment), then ligating the blunt ends together.

\section{Genetic engineering, growth and harvest of cyanobacterial strains}

The seven pAM4418-derived plasmids were used to transform E. coli DH10B cells containing the conjugal and helper plasmids, pRL443 and pRL623, respectively. Transformants were grown overnight in rich LB media, washed with fresh LB, and resuspended in BG-11 media as a tenfold concentrated stock. Cultures of the three host cyanobacteria (Leptolyngbya sp. strain BL0902,
Anabaena sp. PCC7120 in BG-11 media and Synechococcus sp. PCC7002 in Medium A [75]) were grown to late exponential phase, harvested by centrifugation and washed twice with fresh media, before resuspension as a fourfold concentrated stock. Cyanobacterial suspensions were sonicated in a bath for $10 \mathrm{~min}$ to reduce the length of the multicellular strands, then mixed with DH10B transformants. Cell mixtures were centrifuged, resuspended in $200 \mu \mathrm{L}$ of BG-11 media, incubated for $1 \mathrm{~h}$ at $30{ }^{\circ} \mathrm{C}$, then spread on BG-11/5\% LB agar plates. After incubation for $24 \mathrm{~h}$ in low light at $30^{\circ} \mathrm{C}$, cells were washed and spread on BG-11 agar containing $2 \mu \mathrm{g} / \mathrm{mL}$ spectinomycin and streptomycin. After 7-10 days incubation at $30{ }^{\circ} \mathrm{C}$ under illumination $[\sim 20-30 \mu \mathrm{mol}$ photons $\left./\left(\mathrm{m}^{2} \cdot \mathrm{s}\right)\right]$, single colonies were restreaked onto a fresh antibiotic-containing plate and incubated for 5-7 days. Bacteria scraped from the plate were transferred to $30 \mathrm{~mL}$ of BG-11 in a 250-mL conical flask, grown for 5 days at $30{ }^{\circ} \mathrm{C}$ (with shaking at $120 \mathrm{rpm}$ and illumination), then harvested by centrifugation $(5000 \mathrm{rpm}$ for $10 \mathrm{~min}$ ). For Synechococcus sp. PCC7002, Medium A replaced BG-11 media in all steps of the conjugation and growth. Cell pellets were stored frozen at $-80{ }^{\circ} \mathrm{C}$, then dried by lyophilization and weighed before the analysis of lipid content.

\section{Characterization of the fatty acid content of cyanobacteria lipids}

Lyophilized cell pellets were extracted utilizing a modified Bligh/Dyer extraction for total fatty acid analysis ( $2 \mathrm{mg} / \mathrm{sample})$. For total fatty acid analysis, solvents were evaporated under a stream of nitrogen in the presence of a fatty acid internal standard (triheptanoin, $10 \mu \mathrm{g})$. The dried extract was then subjected to base hydrolysis and derivatization in the presence of boron trifluoride $\left(5 \mathrm{~min}, 100{ }^{\circ} \mathrm{C}\right)$ to form fatty acid methyl esters (FAME) following a modification of the protocol by Metcalfe et al. [76] as previously described [77, 78]. FAMEs were analyzed on an Agilent J\&W DB-23 column $(30 \mathrm{~m} \times 0.25 \mathrm{~mm}$ ID, film thickness $0.25 \mu \mathrm{m})$ using HP 5890 gas chromatography (GC) with a flame ionization detector (FID). Individual fatty acids were identified by their elution times relative to authenticated fatty acid standards, and fatty acid quantities were determined by their abundance relative to the internal standard.

For lipidomics analysis, total lipid extracts derived from $2 \mathrm{mg}$ lyophilized biomass from wild type and pDBV-modified strains of Leptolyngbya sp. strain BL0902 and Synechococcus sp. PCC7002 were dried as above and dissolved in $100 \mu \mathrm{L}$ isopropyl alcohol/methanol (50:50) for LC-MS/MS analysis. Samples (10\% of total per injection) were analyzed on a high-resolution $\mathrm{Q}$ Exactive HF Hybrid Quadrupole-Orbitrap Mass Spectrometer 
equipped with a heated electrospray ionization (HESI)-II source (Thermo Scientific, Rockford, IL) and a Vanquish Horizon UHPLC system (Thermo Scientific, Rockford, IL), with source parameters as follows: sheath gas flow rate, $40 \mathrm{~L} / \mathrm{min}$; auxiliary gas flow rate, $5 \mathrm{~L} / \mathrm{min}$; spray voltage, $4.0 \mathrm{kV}$ with positive mode and $3.5 \mathrm{kV}$ with negative mode; capillary temperature, $350{ }^{\circ} \mathrm{C}$; $\mathrm{S}$-lens $\mathrm{RF}$ voltage, $75 \mathrm{~V}$.

Chromatographic separation was achieved on an Accucore C30 column $(2.6 \mu \mathrm{m}, 3 \mathrm{~mm} \times 150 \mathrm{~mm}$, Thermo Scientific, Rockford, IL) with linear gradient elution consisting of mobile phases A (water/ acetonitrile $=40: 60$ ) and B (isopropyl alcohol/acetonitrile $=90: 10$ ) at $0.35 \mathrm{~mL} / \mathrm{min}$. Both mobile phases contained $0.1 \%$ formic acid and $10 \mathrm{mM}$ ammonium formate and the gradient was from $40 \% \mathrm{~B}$ at $0 \mathrm{~min}$ to $95 \%$ $\mathrm{B}$ at $30 \mathrm{~min}$.

MS spectra were acquired by data-dependent scans in positive and negative mode. A survey scan was performed at MS1 level to identify top ten most abundant precursor ions followed by MS2 scans where product ions were generated from selected ions. High-energy collisional dissociation (HCD) was utilized for ion fragmentation with stepped collision energy of $25 / 30 \mathrm{eV}$ and $30 / 50 / 100 \mathrm{eV}$ in each positive and negative polarity [79]. The dynamic exclusion option was enabled during datadependent scans to enhance compound identification in complex mixtures. Acquired spectra were processed using LipidSearch software v4.1 (Thermo Scientific, Rockford, IL) with the selection of following classes of lipids: lysophosphatidylcholine (LPC), phosphatidylcholine (PC), lysophosphatidylethanolamine (LPE), phosphatidylethanolamine (PE), lysophosphatidylserine (LPS), phosphatidylserine (PS), lysophosphatidylglycerol (LPG), phosphatidylglycerol (PG), lysophosphatidylinositol (LPI), phosphatidylinositol (PI), lysophosphatidic acid (LPA), phosphatidic acid (PA), sphingomyelin (SM), phytosphingosine (phSM), monoglyceride (MG), diglyceride (DG), triglyceride (TG), fatty acid (FA), (O-acyl)-1-hydroxy fatty acid (OAHFA), cardiolipin (CL), sphingosine (So), sphingosine phosphate (SoP), glucosylsphingosine (SoG1), monoglycosylceramide (CerG1), diglycosylceramide (CerG2), triglycosylceramide (CerG3), ceramides (Cer), monosialotetrahexosylganglioside (GM2), cholesteryl ester (ChE), zymosteryl $(\mathrm{ZyE})$, stigmasteryl ester (StE), sitosteryl ester (SiE), coenzymes (Co), monogalactosylmonoacylglycerol (MGMG), monogalactosyldiacylglycerol (MGDG), digalactosylmonoacylglycerol (DGMG), digalactosyldiacylglycerol (DGDG), sulfoquinovosylmonoacylglycerol (SQMG), and sulfoquinovosyldiacylglycerol (SQDG). Parameters for the product search workflow were: precursor mass tolerance, $5 \mathrm{ppm}$; product mass tolerance, $5 \mathrm{ppm}$; product ion intensity threshold, $1.0 \%$ relative to precursor; matching score threshold, 2.0. All peak areas were normalized to the total ion current (the total area under the curve in the chromatogram).

\section{Supplementary information}

Supplementary information accompanies this paper at https://doi. org/10.1186/s13068-020-01719-7.

Additional file 1. Supplemental information reporting bioinformatics, as well as additional fatty acid profile and lipidomics data for all three cyanobacterial strains.

Additional file 2. Fatty acid profile for cyanobacteria with and without plasmid constructs.

Additional file 3. Identification of glycoglycerolipids in lipid extracts of wild type and genetically-engineered cyanobacteria.

\section{Abbreviations}

LC: Long chain; FA: Fatty acid; PUFA: Polyunsaturated fatty acid; desB and DesB: $\triangle 15$ Acyl-lipid desaturase (gene and protein, respectively); desD and DesD: $\Delta 6$ Acyl-lipid desaturase (gene and protein, respectively); Vipp1: Vesicle-inducing protein in plastids 1; DesA: $\triangle 12$ Acyl-lipid desaturase; DesC: $\triangle 9$ Acyl-lipid desaturase; SDA: Stearidonic acid (18:4, 18 carbons with 4 double bonds, positions 6, 9, 12, 15); ALA: Alpha-linolenic acid (18:3, double bond positions 9, 12, 15); ETA: Eicosatetraenoic acid (20:4, double bond positions 8, 11, 14, 17); GLA: Gamma-linolenic acid (18:3, double bond positions 6, 9, 12); LA: Linoleic acid (18:2, double bond positions 9, 12); MGDG: Monogalactosyldiacylglycerol; DGDG: Digalactosyldiacylglycerol; SQDG: Sulfoquinovosyldiacylglycerol; PG: Phosphatidylglycerol; pDBV: Plasmid derived from pAM4418 containing genes encoding DesD, DesB and Vipp1; EPA: Eicosapentaenoic acid (20:5, double bond positions 5, 8, 11, 14, 17); DHA: Docosahexaenoic acid (22:6, double bond positions 4, 7, 10, 13, 16, 19); CoA: Coenzyme A; ACP: Acyl carrier protein; BL0902: Leptolyngbya sp. strain BL0902; 7002: Synechococcus sp. PCC7002; 7120: Anabaena sp. PCC7120; GC-FID: Gas chromatography-flame ionization detection; COX1 and COX2: Cyclooxygenases 1 and 2; PCR: Polymerase chain reaction; FAME: Fatty acid methyl esters; LC-MS/MS: Liquid chromatography-tandem mass spectrometry; UHPLC: Ultrahigh performance liquid chromatography.

\section{Acknowledgements}

We are grateful to Dr. James Golden (University of California at San Diego) for providing the pAM4418 plasmid and the strains of cyanobacteria tested in this work, as well as advice regarding growth of cyanobacteria. We thank Deena Wykle for assistance with literature searches.

\section{Authors' contributions}

LBP, DP and FHC conceived of and designed the approaches and plasmid constructs. DP, SS and LRM conducted experiments and collected data. JL performed the mass spectrometry experiments. Data analysis and interpretation, well as manuscript preparation and editing, was performed by LBP, DP, SS $J$, CMF and FHC. LBP and FHC were responsible for the coordination of the work. All authors read and approved the final manuscript.

\section{Funding}

We thank Wake Forest Innovations for financial support through the Value Inflection, Commercialization Pathway and Catalyst Awards, and particularly Sarah Haigh Molina for her efforts in supporting this work. We also acknowledge contributions from the National Institutes of Health, R01 AT008621 (to FHC), as well as R01 GM119227 and R35 GM135179 (to LBP). We also acknowledge the support provided by the Proteomics and Metabolomics Shared Resource of the Wake Forest Baptist Comprehensive Cancer Center ( $\mathrm{NIH} / \mathrm{NCl}$ P30 CA12197; facility Director Dr. Cristina M. Furdui).

Availability of data and materials

All data generated or analyzed during this study are included in this published article and its additional files. 
Ethics approval and consent to participate

Not applicable.

\section{Consent for publication}

Not applicable.

\section{Competing interests}

LBP, FHC, DP and SS are coinventors on a Patent Cooperation Treaty (PCT) patent application (PCT/US17/52938), which claims priority to provisional patent application 62/398,604.

\section{Author details}

${ }^{1}$ Department of Biochemistry, Wake Forest School of Medicine, Winston-Salem, NC 27157, USA. ${ }^{2}$ Center for Redox Biology and Medicine, Wake Forest School of Medicine, Winston-Salem, NC 27157, USA. ${ }^{3}$ Department of Physiology and Pharmacology, Wake Forest School of Medicine, Winston-Salem, NC 27157, USA. ${ }^{4}$ Department of Internal Medicine, Section on Molecular Medicine, Wake Forest School of Medicine, Winston-Salem, NC 27157, USA. ${ }^{5}$ Comprehensive Cancer Center, Wake Forest School of Medicine, Winston-Salem, NC 27157, USA. ${ }^{6}$ Department of Nutritional Sciences and the $\mathrm{BIO} 5$ Institute, University of Arizona, Tucson, AZ, USA. ${ }^{7}$ Present Address: 139 N St. Patrick St., New Orleans, LA 70119, USA.

Received: 12 January 2020 Accepted: 16 April 2020

Published online: 06 May 2020

\section{References}

1. Mozaffarian D, Wu JH. Omega-3 fatty acids and cardiovascular disease: effects on risk factors, molecular pathways, and clinical events. J Am Coll Cardiol. 2011;58:2047-67.

2. Campoy C, Escolano-Margarit MV, Anjos T, Szajewska H, Uauy R. Omega 3 fatty acids on child growth, visual acuity and neurodevelopment. Br J Nutr. 2012;107(Suppl 2):S85-106.

3. Grosso G, Galvano F, Marventano S, Malaguarnera M, Bucolo C, Drago F, Caraci F. Omega-3 fatty acids and depression: scientific evidence and biological mechanisms. Oxid Med Cell Longev. 2014;2014:313570.

4. Grosso G, Pajak A, Marventano S, Castellano S, Galvano F, Bucolo C, Drago F, Caraci F. Role of omega-3 fatty acids in the treatment of depressive disorders: a comprehensive meta-analysis of randomized clinical trials. PLOS ONE. 2014;9:e96905.

5. Luchtman DW, Song C. Cognitive enhancement by omega-3 fatty acids from child-hood to old age: findings from animal and clinical studies. Neuropharmacology. 2013;64:550-65.

6. Adarme-Vega TC, Thomas-Hall SR, Schenk PM. Towards sustainable sources for omega-3 fatty acids production. Curr Opin Biotechnol. 2014;26:14-8.

7. Finco AMO, Mamani LDG, Carvalho JC, de Melo Pereira GV, ThomazSoccol V, Soccol CR. Technological trends and market perspectives for production of microbial oils rich in omega-3. Crit Rev Biotechnol. 2017;37:656-71.

8. Carmona-Antonanzas G, Tocher DR, Martinez-Rubio L, Leaver MJ. Conservation of lipid metabolic gene transcriptional regulatory networks in fish and mammals. Gene. 2014;534:1-9.

9. Leaver MJ, Villeneuve LA, Obach A, Jensen L, Bron JE, Tocher DR, Taggart JB. Functional genomics reveals increases in cholesterol biosynthetic genes and highly unsaturated fatty acid biosynthesis after dietary substitution of fish oil with vegetable oils in Atlantic salmon (Salmo salar). BMC Genomics. 2008;9:299.

10. Bell JG, Tocher DR, Henderson RJ, Dick JR, Crampton VO. Altered fatty acid compositions in atlantic salmon (Salmo salar) fed diets containing linseed and rapeseed oils can be partially restored by a subsequent fish oil finishing diet. J Nutr. 2003;133:2793-801.

11. Monge-Ortiz R, Tomás-Vidal A, Rodriguez-Barreto D, Martínez-Llorens S, Pérez JA, Jover-Cerdá M, Lorenzo A. Replacement of fish oil with vegetable oil blends in feeds for greater amberjack (Seriola dumerili) juveniles: effect on growth performance, feed efficiency, tissue fatty acid composition and flesh nutritional value. Aquac Nutr. 2018;24:605-15.

12. Ma X, Jiang Z, Lai C. Significance of Increasing n-3 PUFA Content in Pork on Human Health. Crit Rev Food Sci Nutr. 2016;56:858-70.
13. Lee SA, Whenham N, Bedford MR. Review on docosahexaenoic acid in poultry and swine nutrition: consequence of enriched animal products on performance and health characteristics. Anim Nutr. 2019;5:11-21.

14. James MJ, Ursin VM, Cleland LG. Metabolism of stearidonic acid in human subjects: comparison with the metabolism of other n-3 fatty acids. Am J Clin Nutr. 2003;77:1140-5.

15. Surette ME, Edens M, Chilton FH, Tramposch KM. Dietary echium oil increases plasma and neutrophil long-chain (n-3) fatty acids and lowers serum triacylglycerols in hypertriglyceridemic humans. J Nutr. 2004;134:1406-11.

16. Whelan J. Dietary stearidonic acid is a long chain ( $n-3)$ polyunsaturated fatty acid with potential health benefits. J Nutr. 2009;139:5-10.

17. Elkin RG, Ying Y, Harvatine KJ. Feeding laying hens stearidonic acidenriched soybean oil, as compared to flaxseed oil, more efficiently enriches eggs with very long-chain n-3 polyunsaturated fatty acids. J Agric Food Chem. 2015;63:2789-97.

18. Ursin VM. Modification of plant lipids for human health: development of functional land-based omega-3 fatty acids. J Nutr. 2003;133:4271-4.

19. Eckert H, La Vallee B, Schweiger BJ, Kinney AJ, Cahoon EB, Clemente T. Coexpression of the borage Delta 6 desaturase and the Arabidopsis Delta 15 desaturase results in high accumulation of stearidonic acid in the seeds of transgenic soybean. Planta. 2006;224:1050-7.

20. Harris WS. The omega-3 index as a risk factor for coronary heart disease. Am J Clin Nutr. 2008;87:1997S-2002S.

21. Lemke SL, Vicini JL, Su H, Goldstein DA, Nemeth MA, Krul ES, Harris WS. Dietary intake of stearidonic acid-enriched soybean oil increases the omega-3 index: randomized, double-blind clinical study of efficacy and safety. Am J Clin Nutr. 2010;92:766-75

22. Thajuddin N, Subramanian G. Cyanobacterial biodiversity and potential applications in biotechnology. Curr Sci. 2005;89:47-57.

23. Gomaa MA, Al-Haj L, Abed RM. Metabolic engineering of Cyanobacteria and microalgae for enhanced production of biofuels and high-value products. J Appl Microbiol. 2016;121:919-31.

24. Radakovits R, Jinkerson RE, Darzins A, Posewitz MC. Genetic engineering of algae for enhanced biofuel production. Eukaryot Cell. 2010;9:486-501.

25. Liu X, Miao R, Lindberg P, Lindblad P. Modular engineering for efficient photosynthetic biosynthesis of 1-butanol from $\mathrm{CO}_{2}$ in cyanobacteria. Energy Environ Sci. 2019;12:2765-77.

26. Liu X, Sheng J, Curtiss R 3rd. Fatty acid production in genetically modified cyanobacteria. Proc Natl Acad Sci USA. 2011;108:6899-904.

27. Ruffing AM, Jones HD. Physiological effects of free fatty acid production in genetically engineered Synechococcus elongatus PCC 7942. Biotechnol Bioeng. 2012;109:2190-9.

28. Ng IS, Keskin BB, Tan SI. A critical review of genome editing and synthetic biology applications in metabolic engineering of microalgae and cyanobacteria. Biotechnol J. 2020:e1900228.

29. Pittera J, Jouhet J, Breton S, Garczarek L, Partensky F, Marechal E, Nguyen NA, Dore H, Ratin M, Pitt FD, Scanlan DJ, Six C. Thermoacclimation and genome adaptation of the membrane lipidome in marine Synechococcus. Environ Microbiol. 2018;20:612-31.

30. Sakamoto T, Los DA, Higashi S, Wada H, Nishida I, Ohmori M, Murata $\mathrm{N}$. Cloning of omega 3 desaturase from cyanobacteria and its use in altering the degree of membrane-lipid unsaturation. Plant Mol Biol. 1994;26:249-63.

31. Kagan ML, West AL, Zante C, Calder PC. Acute appearance of fatty acids in human plasma - a comparative study between polar-lipid rich oil from the microalgae Nannochloropsis oculata and krill oil in healthy young males. Lipids Health Dis. 2013;12:102.

32. Dyerberg J, Madsen P, Moller JM, Aardestrup I, Schmidt EB. Bioavailability of marine n-3 fatty acid formulations. Prostaglandins Leukot Essent Fatty Acids. 2010;83:137-41.

33. Sugawara T, Miyazawa T. Separation and determination of glycolipids from edible plant sources by high-performance liquid chromatography and evaporative light-scattering detection. Lipids. 1999;34:1231-7.

34. Murata N, Wada H. Acyl-lipid desaturases and their importance in the tolerance and acclimatization to cold of cyanobacteria. Biochem J. 1995;308(Pt 1):1-8.

35. Andersson L, Carriere F, Lowe ME, Nilsson A, Verger R. Pancreatic lipaserelated protein 2 but not classical pancreatic lipase hydrolyzes galactolipids. Biochim Biophys Acta. 1996;1302:236-40. 
36. Kagan ML, Levy A, Leikin-Frenkel A. Comparative study of tissue deposition of omega-3 fatty acids from polar-lipid rich oil of the microalgae Nannochloropsis oculata with krill oil in rats. Food Funct. 2015;6:186-92.

37. Los DA, Mironov KS. Modes of Fatty Acid desaturation in cyanobacteria: an update. Life. 2015;5:554-67.

38. Kobayashi K, Endo K, Wada H. Specific Distribution of Phosphatidylglycerol to Photosystem Complexes in the Thylakoid Membrane. Front Plant Sci. 2017;8:1991.

39. Tasaka Y, Gombos Z, Nishiyama Y, Mohanty P, Ohba T, Ohki K, Murata N. Targeted mutagenesis of acyl-lipid desaturases in Synechocystis: evidence for the important roles of polyunsaturated membrane lipids in growth, respiration and photosynthesis. EMBO J. 1996;15:6416-25.

40. Chi X, Yang Q, Zhao F, Qin S, Yang Y, Shen J, Lin H. Comparative analysis of fatty acid desaturases in cyanobacterial genomes. Comp Funct Genomics. 2008:284508.

41. Wang M, Chen H, Gu Z, Zhang H, Chen W, Chen YQ. Omega3 fatty acid desaturases from microorganisms: structure, function, evolution, and biotechnological use. Appl Microbiol Biotechnol. 2013;97:10255-62.

42. Los DA, Murata N. Structure and expression of fatty acid desaturases. Biochim Biophys Acta. 1998;1394:3-15.

43. Heidrich J, Thurotte A, Schneider D. Specific interaction of IM30/Nipp1 with cyanobacterial and chloroplast membranes results in membrane remodeling and eventually in membrane fusion. Biochim Biophys Acta Biomembr. 2017;1859:537-49.

44. Taton A, Lis E, Adin DM, Dong G, Cookson S, Kay SA, Golden SS, Golden JW. Gene transfer in Leptolyngbya sp strain BL0902, a cyanobacterium suitable for production of biomass and bioproducts. PLoS ONE. 2012; 7:ee30901.

45. Taton A, Unglaub F, Wright NE, Zeng WY, Paz-Yepes J, Brahamsha B, Palenik B, Peterson TC, Haerizadeh F, Golden SS, Golden JW. Broadhost-range vector system for synthetic biology and biotechnology in cyanobacteria. Nucleic Acids Res. 2014;42:e136.

46. Chen G, Qu S, Wang Q, Bian F, Peng Z, Zhang Y, Ge H, Yu J, Xuan N, Bi Y, He Q. Transgenic expression of delta- 6 and delta- 15 fatty acid desaturases enhances omega-3 polyunsaturated fatty acid accumulation in Synechocystis sp. PCC6803. Biotechnol Biofuels. 2014;7:32.

47. Yoshino T, Kakunaka N, Liang Y, Ito Y, Maeda Y, Nomaguchi T, Matsunaga T, Tanaka T. Production of omega 3 fatty acids in marine cyanobacterium Synechococcus sp. strain NKBG 15041c via genetic engineering. Appl Microbiol Biotechnol. 2017;101:6899-905.

48. Kroll D, Meierhoff K, Bechtold N, Kinoshita M, Westphal S, Vothknecht UC, Soll J, Westhoff P. VIPP1, a nuclear gene of Arabidopsis thaliana essential for thylakoid membrane formation. Proc Natl Acad Sci USA. 2001;98:4238-42.

49. Bohuszewicz O, Liu J, Low HH. Membrane remodelling in bacteria. J Struct Biol. 2016;196:3-14.

50. Los DA, Ray MK, Murata N. Differences in the control of the temperaturedependent expression of four genes for desaturases in Synechocystis sp. PCC 6803. Mol Microbiol. 1997;25:1167-75.

51. Sakamoto T, Bryant DA. Temperature-regulated mRNA accumulation and stabilization for fatty acid desaturase genes in the cyanobacterium Synechococcus sp strain PCC 7002. Mol Microbiol. 1997:23:1281-92.

52. Sakamoto T, Higashi S, Wada H, Murata N, Bryant DA. Low-temperatureinduced desaturation of fatty acids and expression of desaturase genes in the cyanobacterium Synechococcus sp. PCC 7002. FEMS Microbiol Lett. 1997;152:313-20

53. Cohen Z, Vonshak A, Richmond A. Fatty acid composition of Spirulina strains grown under various environmental conditions. Phytochemistry. 1987;26:2255-8.

54. Santos-Merino M, Garcillan-Barcia MP, de la Cruz F. Engineering the fatty acid synthesis pathway in Synechococcus elongatus PCC 7942 improves omega-3 fatty acid production. Biotechnol Biofuels. 2018;11:239.

55. Hammond BG, Lemen JK, Ahmed G, Miller KD, Kirkpatrick J, Fleeman T. Safety assessment of SDA soybean oil: results of a 28-day gavage study and a 90-day/one generation reproduction feeding study in rats. Regul Toxicol Pharmacol. 2008;52:311-23.

56. Guedes AC, Amaro HM, Barbosa CR, Pereira RD, Malcata FX. Fatty acid composition of several wild microalgae and cyanobacteria, with a focus on eicosapentaenoic, docosahexaenoic and alpha-linolenic acids for eventual dietary uses. Food Res Int. 2011;44:2721-9.

57. Ruiz-Lopez N, Haslam RP, Venegas-Caleron M, Larson TR, Graham IA, Napier JA, Sayanova O. The synthesis and accumulation of stearidonic acid in transgenic plants: a novel source of 'heart-healthy' omega-3 fatty acids. Plant Biotechnol J. 2009;7:704-16.

58. Coupland K. Stearidonic acid: a plant produced omega-3 PUFA and a potential alternative for marine oil fatty acids. Lipid Technology. 2008;20:152-4.

59. Zhao X-R, Robert S, Singh S, Green A. Heterologous production of GLA and SDA by expression of an Echium plantagineum $\triangle 6$-desaturase gene. Plant Sci. 2006;170:665-73.

60. Ruiz-Lopez N, Haslam RP, Usher S, Napier JA, Sayanova O. An alternative pathway for the effective production of the omega-3 long-chain polyunsaturates EPA and ETA in transgenic oilseeds. Plant Biotechnol J. 2015;13:1264-75

61. Treschow AP, Hodges LD, Wright PF, Wynne PM, Kalafatis N, Macrides TA. Novel anti-inflammatory omega-3 PUFAs from the New Zealand greenlipped mussel, Perna canaliculus. Comp Biochem Physiol B: Biochem Mol Biol. 2007; 147:645-56.

62. Grienke U, Silke J, Tasdemir D. Bioactive compounds from marine mussels and their effects on human health. Food Chem. 2014;142:48-60.

63. McPhee S, Hodges LD, Wright PF, Wynne PM, Kalafatis N, Harney DW, Macrides TA. Anti-cyclooxygenase effects of lipid extracts from the New Zealand green-lipped mussel, Perna canaliculus. Comp Biochem Physiol B: Biochem Mol Biol. 2007;146:346-56.

64. Wilson DB, Prescott SM, Majerus PW. Discovery of an arachidonoyl coenzyme A synthetase in human platelets. J Biol Chem. 1982;257:3510-5.

65. Emelyanov A, Fedoseev G, Krasnoschekova O, Abulimity A, Trendeleva T, Barnes PJ. Treatment of asthma with lipid extract of New Zealand greenlipped mussel: a randomised clinical trial. Eur Respir J. 2002;20:596-600.

66. Roulet J, Taton A, Golden JW, Arabolaza A, Burkart MD, Gramajo H. Development of a cyanobacterial heterologous polyketide production platform. Metab Eng. 2018;49:94-104.

67. Forrest LM, Lough CM, Chung S, Boudyguina EY, Gebre AK, Smith TL, Colvin PL, Parks JS. Echium oil reduces plasma triglycerides by increasing intravascular lipolysis in apoB100-only low density lipoprotein (LDL) receptor knockout mice. Nutrients. 2013;5:2629-45.

68. Deckelbaum RJ, Calder PC, Harris WS, Akoh CC, Maki KC, Whelan J, Banz WJ, Kennedy E. Conclusions and recommendations from the symposium, Heart Healthy Omega-3 s for Food: Stearidonic Acid (SDA) as a Sustainable Choice. J Nutr. 2012;142:641S-3S.

69. Harris WS, Lemke SL, Hansen SN, Goldstein DA, DiRienzo MA, Su H, Nemeth MA, Taylor ML, Ahmed G, George C. Stearidonic acid-enriched soybean oil increased the omega-3 index, an emerging cardiovascular risk marker. Lipids. 2008:43:805-11.

70. Krul ES, Lemke SL, Mukherjea R, Taylor ML, Goldstein DA, Su H, Liu P, Lawless A, Harris WS, Maki KC. Effects of duration of treatment and dosage of eicosapentaenoic acid and stearidonic acid on red blood cell eicosapentaenoic acid content. Prostaglandins Leukot Essent Fatty Acids. 2012;86:51-9.

71. Elkin RG, Ying Y, Fan Y, Harvatine KJ. Influence of feeding stearidonic acid (18:4n-3)-enriched soybean oil, as compared to conventional soybean oil, on tissue deposition of very long-chain omega-3 fatty acids in meat-type chickens. Anim Feed Sci Technol. 2016;217:1-12.

72. Kitessa SM, Young P. Enriching milk fat with $n-3$ polyunsaturated fatty acids by supplementing grazing dairy cows with ruminally protected Echium oil. Anim Feed Sci Technol. 2011;170:35-44.

73. Albert BB, Derraik JG, Cameron-Smith D, Hofman PL, Tumanov S, VillasBoas SG, Garg ML, Cutfield WS. Fish oil supplements in New Zealand are highly oxidised and do not meet label content of n-3 PUFA. Sci Rep. 2015;5:7928.

74. Kolanowski W. Omega-3 LC PUFA contents and oxidative stability of encapsulated fish oil dietary supplements. Int J Food Prop. 2010;13:498-511.

75. Stevens SE, Patterson CO, Myers J. The production of hydrogen peroxide by blue-green algae: a survey. J Phycol. 1973;9:427-30. 
76. Metcalfe LD, Schmitz AA, Pelka JR. Rapid preparation of fatty acid esters from lipids for gas chromatographic analysis. Anal Chem. 1966;38:514-5.

77. Weaver KL, Ivester P, Seeds MC, Case LD, Arm J, Chilton FH. Effect of dietary fatty acids on inflammatory gene expression in healthy humans. J Biol Chem. 2009;284:15400-7.

78. Sergeant S, Hugenschmidt CE, Rudock ME, Ziegler JT, Ivester P, Ainsworth HC, Vaidya D, Case LD, Langefeld CD, Freedman BI, Bowden DW, Mathias RA, Chilton FH. Differences in arachidonic acid levels and fatty acid desaturase (FADS) gene variants in African Americans and European Americans with diabetes or the metabolic syndrome. Br J Nutr. 2012;107:547-55.

79. Bird SS, Marur VR, Sniatynski MJ, Greenberg HK, Kristal BS. Lipidomics profiling by high-resolution LC-MS and high-energy collisional dissociation fragmentation: focus on characterization of mitochondrial cardiolipins and monolysocardiolipins. Anal Chem. 2011;83:940-9.

80. Dong X, He Q, Peng Z, Yu J, Bian F, Li Y, Bi Y. Production of Y-linolenic acid and stearidonic acid by Synechococcus sp. PCC7002 containing cyanobacterial fatty acid desaturase genes. Chin J Ocean Limn. 2016;34:772-80.

\section{Publisher's Note}

Springer Nature remains neutral with regard to jurisdictional claims in published maps and institutional affiliations.
Ready to submit your research? Choose BMC and benefit from:

- fast, convenient online submission

- thorough peer review by experienced researchers in your field

- rapid publication on acceptance

- support for research data, including large and complex data types

- gold Open Access which fosters wider collaboration and increased citations

- maximum visibility for your research: over $100 \mathrm{M}$ website views per year

At BMC, research is always in progress.

Learn more biomedcentral.com/submissions 